\title{
A Dead Sea Water-Enriched Body Cream Improves Skin Severity Scores in Children with Atopic Dermatitis
}

\author{
Meital Portugal-Cohen ${ }^{1 *}$, Miriam Oron ${ }^{1}$, Efrat Merrik², Ze'evi Ma'or ${ }^{1}$, Dan Ben-Amitai ${ }^{2}$, \\ Hagai Yogev $^{3}$, Alex Zvulunov ${ }^{2,4}$
}

\begin{abstract}
${ }^{1}$ AHAVA-Dead Sea Laboratories, and Dead Sea and Arava Science Center, Dead Sea, Israel; ${ }^{2}$ Pediatric Dermatology Unit, Schneider Children's Medical Center, Petah-Tiqva, Israel; ${ }^{3}$ Tel Aviv-Yaffo Academic College, Tel Aviv, Israel; ${ }^{4}$ Ben-Gurion University of the Negev, Beer-Sheva, Israel.

Email:*meital.p@ahava.com; meitalp@ekmd.huji.ac.il
\end{abstract}

Received June $28^{\text {th }}, 2011$; revised July $18^{\text {th }}, 2011$; accepted July $29^{\text {th }}, 2011$.

\begin{abstract}
*
Dead Sea (DS) mud and water are known for their unique composition of minerals, and for their therapeutic properties on inflammatory skin diseases. The objective of the study was to evaluate the efficacy of an emollient cream enriched with $D S$ water in children with atopic dermatitis $(A D)$. Eighty six $A D$ children were randomized in a double-blind controlled study to receive twice-daily topical treatment with a body cream enriched with DS minerals (TP) compared to two types of control: 1) DM, DS minerals with lower DS water concentrations than TP, and 2) an emollient (E) with no DS minerals. Efficacy was assessed by a change in clinical skin severity scores: SCORing Atopic Dermatitis (SCORAD), investigator's global assessment (IGA) and patient global assessment (PGA) as well as by objective physiological parameters: transepidermal water loss (TEWL), stratum corneum hydration (SCH), affected body surface area (BSA) and Objective Severity Assessment of Atopic Dermatitis (OSAAD). The total length of the trial was 12 weeks divided to 6 visits at weeks $0,2,4,6,8,12$. The study showed that both TP and DM creams improved OSAAD scores. Only TP improved TEWL and SCH. TP was the most effective regarding TEWL, SCH and OSAAD compared to DM and E. Treatment with E decreased more significantly IGA score compared to TP. Although within each treatment group significant improvements in SCH, BSA, SCORAD, IGA and PGA were observed, the reduction in BSA, SCORAD and PGA was not significantly different among the groups. Our results clearly show the benefits of TP as a leave on-skin emulsion enrich with DS water in terms of skin barrier function. Thus, TP can serve as an effective adjuvant treatment for AD skin as well as for its maintenance.
\end{abstract}

Keywords: Atopic Dermatitis, Dead Sea Minerals, Skin Barrier Function, Leave-on Skin Preparation

\section{Introduction}

Atopic dermatitis (AD) is a frequent Th2-mediated inflammatory disease, which occurs in persons of all ages but is more common in children, with the prevalence rate of around $10 \%$ to $20 \%$. Its chronically relapsing course is influenced by local, immunological, genetic and environmental factors [1-3]. Dry skin, intense pruritus, impaired epidermal barrier function and a susceptibility to cutaneous infections are major features of $\mathrm{AD}[4,5]$.

Topical treatments focus on reducing both the dryness and inflammation of the skin. Daily application of moisturizing emollients is crucial to improve dry and inflamed skin [4,6-8].
The beneficial effects of Dead Sea (DS) climathotherapy in inflammatory skin disorders had been repeatedly demonstrated. [9-11]. DS minerals (i.e. DS mud and DS water) composition is especially rich in magnesium, calcium, sodium, potassium, zinc, strontium, sulphides and bromides $[12,13]$. Some of these minerals are known to influence signal transduction and cell metabolism: it has been demonstrated that $\mathrm{Mg}^{2+}$ ions inhibit the antigen-presenting function of human epidermal Langerhans cells in vivo and in vitro $[14,15]$. Bathing in a magnesium-rich Dead Sea salt solution improves skin barrier function, enhances skin hydration, and reduces inflamemation in atopic dry skin [16]. Zinc is involved in epidermal proliferation and wound healing [17]. The thera- 
peutic properties of the mud are ascribed to its content of reduced sulphur species, its physical and chemical properties and its mineral content [18]. Therapeutic value of DS minerals, reported on various inflammatory skin disorders, such as psoriasis, contact and atopic dermatitis, might be enhanced by easier trans-epidermal penetration in lesional areas, due to stratum corneum damage [19]. Alternatively, limited penetration may be enough to activate signaling pathways by receptor binding $[17,20]$, or even non-binding activation may occur [21].

Dermud $^{\mathrm{TM}}$ cream $(\mathrm{DM})$ and Clineral Topic ${ }^{\mathrm{TM}}$ Body cream (TP) were formulated as a cosmeceutical compositions by Ahava-Dead Sea Laboratories Ltd and patented by Dead Sea Laboratories LTD (US patent 6582689).

$\mathrm{DM}$ is a commercial product, especially designed for dry and sensitive, but otherwise healthy skin and contains $1.9 \%$ Dead Sea mud and 1\% Dead Sea water. Previous studies revealed that DM has protective, anti-oxidant and anti-inflammatory properties that can antagonize biological effects of UVB irradiation in skin explants [22]. Based on its beneficial effects on normal skin, the need to develop a cream containing DS minerals for atopic disordered skin had been arisen.

Clineral Topic ${ }^{\mathrm{TM}}$ Body cream (TP), was specially designed to alleviate symptoms related to AD. It contains $1.8 \%$ DS mud and $2.5 \%$ Dead Sea water and hence, contains a higher 2.5-fold content of DS water than to DM. Significant improvement of clinical inflammatory signs skin barrier function was lately reported in patients with $\mathrm{AD}$ following bathing in DS water [16]. Therefore, we hypothesized that DS water may be valuable in the treatment of $\mathrm{AD}$. The aim of the present study was to investigate the safety and efficacy of topical application with TP versus DM and versus an emollient control cream with no DS minerals but with active ingredients (E) in the treatment of $\mathrm{AD}$ in children.

\section{Methods}

\subsection{Study Design}

The present study was a randomized controlled double blind trial carried out between July 2005 to December 2007 and between May 2008 to July 2008 in the Pediatric Dermatology Unit, Schneider Children's Medical Center, Israel.

Efficacy was assessed by a change in objective and subjective severity assessment parameters of $\mathrm{AD}$. The total length of the trial was 12 weeks divided to 6 visits at weeks $0,2,4,6,8,12$. Assessment of all efficacy scales performed at visits $1-6$ and was documented and defined as baseline at visit 1 (week 0 ).

The study was presented and approved by the Institutional and Ministry of Health Ethics Committee, approval number 920050440 .

\subsection{Study Population}

Eighty six children aged 2 to10 years with mild or moderate $\mathrm{AD}$ were enrolled to the study. The diagnosis of $\mathrm{AD}$ has been established using modified Hanifin-Rajka criteria [23]. The patients had no other significant concurrent illness and no known allergy to ingredients of the tested creams. Controlled use of topical corticosteroids and oral administration was allowed. The amount of topical corticosteroids had been recorded on each visit.

\subsection{Treatment Regimen}

After meeting all inclusion criteria, subjects were randomized to one of three treatment groups. The patients were instructed to apply the assigned treatment assignment to the whole body skin area (BSA) twice daily (morning and evening) after bathing.

All treatments used in this study were manufactured by AHAVA Dead Sea Laboratories, Ltd., Israel. All three treatments were non-fragranced, oil in water stable emulsions with a $\mathrm{pH}=7$. In two groups: $\mathrm{TP}$ and $\mathrm{DM}$ the applied emollient cream contained DS water and DS mud.

DS water: the major constitutes of DS water are the following ions: $\mathrm{Mg}^{2+}(92,500 \mathrm{mg} / \mathrm{l}), \mathrm{Ca}^{2+}(38,000 \mathrm{mg} / \mathrm{l})$, $\mathrm{K}^{+}(1400 \mathrm{mg} / \mathrm{l}), \mathrm{Na}^{+}(2000 \mathrm{mg} / \mathrm{ml}), \mathrm{Sr}^{2+}(800 \mathrm{mg} / \mathrm{l}), \mathrm{Cl}^{-}$ $(345,000 \mathrm{mg} / \mathrm{l}), \mathrm{Br}^{-}(11,500 \mathrm{mg} / \mathrm{l})$.

DS mud: the major constitutes of DS mud are the following ions: $\mathrm{Mg}^{2+}(35 \mathrm{~g} / \mathrm{l}), \mathrm{Ca}^{2+}(22.5 \mathrm{~g} / \mathrm{l}), \mathrm{K}^{+}(7 \mathrm{~g} / \mathrm{l})$, $\mathrm{Na}^{+}(27 \mathrm{mg} / \mathrm{ml}), \mathrm{Cl}^{-}(174.5 \mathrm{~g} / \mathrm{l})$.

$E$ : a control emollient that does not contain DS minerals, but does include ingredients that have a soothing effect on skin. It contains Lactic Acid, Ethylhexyl Palmitate, Glycerin Dimethicone, and Propylene Glycol as emollients; PEG-40 Stearate, Cetearyl Alcohol (and) Ceteareth-30, Glyceryl Stearate, Sorbitan Tristearate and Cetyl Alcohol as emulsifiers; Additionally, the product contains Aloe Barbadensis Leaf Extract, and Allantoin as anti-irritants. Triclosan is an anti-fungal and anti-bacterial compound, incorporated as a disinfectant for the skin and Vitamin $\mathrm{E}$ as an antioxidant. The preservative system is a mixture of Butyl Paraben, Propyl Paraben, Methyl Paraben, Ethyl Paraben, Isobutylparaben, and Phenoxyethanol.

$D M$ : DM contains DS water and DS mud. It also contains Lactic Acid, Ethylhexyl Palmitate, Glycerin Dimethicone, and Propylene Glycol as emollients; PEG-40 Stearate, Cetearyl Alcohol (and) Ceteareth-30, Glyceryl Stearate, Sorbitan Tristearate and Cetyl Alcohol as emulsifiers; Additionally, the product contains Zinc Oxide, Aloe Barbadensis Leaf Extract, and Allantoin as antiirritants. Triclosan is an anti-fungal and anti-bacterial compound, incorporated as a disinfectant for the skin and 
vitamin $\mathrm{E}$ as an antioxidant. The preservative system is a mixture of Butyl Paraben, Propyl Paraben, Methyl Paraben, Ethyl Paraben, Isobutylparaben, and Phenoxyethanol.

TP: TP contains DS water and enriched with DS mud. It also contains Lactic Acid, Grapefruit seed oil. Ethylhexyl Palmitate, Glycerin, Propanediol and Dimethicone as emollients; PEG-40 Stearate, Cetearyl Alcohol, Ceteareth-30, Glyceryl Stearate, Sorbitan Tristearate and Cetyl Alcohol as emulsifiers; Additionally, the product contains Zinc Oxide, Aloe Barbadensis Leaf Extract, Bisabolol, Hippophae oil, Calendula Officinalis Flower Extract and Allantoin as anti-irritants and Dipotassium Glycyrrhizinate as an anti-inflammatory. Xanthan Gum is a thickener and Vitamin E acetate an antioxidant. The preservative system is a mixture of potassium sorbate, 1,2-Hexanediol and Caprylyl glycol.

\subsection{Blinding}

This was a double blind, controlled study. DM, TP, or E creams were identical in color, texture and scent. All were packaged in containers void of labeling except for the treatment code number and were identical in terms of shape, size and color so that identification of treatment assignment was unknowable to the participant, study investigators and medical personnel.

\subsection{Outcome Measures}

The efficacy assessment parameters were determined as a change from baseline scores of the following variables:

Transepidermal water loss (TEWL) was measured with the open chamber system (Tewameter TM 300, Courage \& Khazaka, Koln, Germany) on uninvolved, mildly involved and severely involved skin sites for each patient. To avoid anatomical differences, the flexor surface of the forearm was used as the measuring site on uninvolved, mildly involved and severely involved skin whenever possible. All patients were acclimatized in the study room for at least $15 \mathrm{~min}$ before the measurements. The evaluation of skin physiology variables for each patient was performed under same room temperature and humidity conditions. The patients were instructed not to apply any topical medication or emollients within $4 \mathrm{~h}$ preceding the measurements. Thirty successive measurements were performed during 30 seconds period at each site and the mean values obtained, expressed in $\mathrm{g} \cdot \mathrm{m}^{-2} \cdot \mathrm{h}^{-1}[24]$.

Stratum corneum hydration $(\mathrm{SCH})$ was assessed by measuring capacitance (Corneometer 825, Courage \& Khazaka, Koln, Germany) on the same uninvolved, mildly involved and severely involved sites for each study subject. Three measurements were performed at each site and the average values, expressed as arbitrary units (AU) were recorded [25].

The body surface area (BSA) of unaffected, mildly affected and severely affected skin was estimated using a computer-assisted method based on OSAAD software (Courage \& Khazaka, Koln, Germany).

OSAAD-Objective Severity Assessment of Atopic Dermatitis score is derived from measurements of stratum corneum barrier function and $\mathrm{SCH}$ and normalized for extent of disease [8]. The OSAAD score was calculated for each study subject on the basis of measurements of TEWL, SCH and computer assisted estimation of BSA. The calculation of OSAAD provides an objective continuous numerical index and its values may range from 0 to 100 [6].

SCORAD-SCORing Atopic Dermatitis was an index of severity derived from measurement of affected skin area and assessment of erythema, edema, oozing or crusting, excoriations, dryness and subjective severity of pruritus and sleep loss.

SCORAD values may range from 0 (no disease) to 103 (whole skin surface is maximally affected). All estimates were performed by the same investigator (AZ).

The SCORAD index was calculated for each patient by estimation of the intensity and extent of affected skin, and subjective symptoms such as sleep loss and pruritus according to the published guidelines 11 [26].

IGA-Investigator's Global Assessment had been rated on every visit on a visual analogue scale $(0-10)$.

PGA-Patient Global Assessment had been rated by the patient's parents on every visit on a visual analogue scale $(0-10)$.

Efficacy was evaluated by a change in SCORAD and OSAAD scores, TEWL and $\mathrm{SCH}$, as well as PGA and IGA.

\subsection{Adverse Events and Tolerance}

All adverse events were recorded and compared by treatment assignment.

Previous clinical trials that were performed independently in order to examine tolerance of DM and TP indicated that these treatments are safe for use.

\subsection{Data Analysis}

The statistical analysis using paired $t$-test for the differences in the subjective and objective variables before and after treatments was performed with SPSS12 software (SPSS Software, Chicago, IL, USA). The comparison was between $\mathrm{TP}$ and $\mathrm{E}$ as well as between TP and MD.

\section{Results}

86 children with AD were recruited for the study, among them 7 dropped out after the first visit due to stinging 
upon application the cream: 1 in TP group, 4 in DM group and 2 in E group. Of the remaining 79 patients, 55 (72\%) completed the study protocol: $11 / 13(85 \%)$ in the study group of TP, 16/26 (62\%) in the control group of DM and 28/40 (70\%), in the control group of E.

A summary of the remaining 79 patients' assessment parameters at the baseline is presented at Table 1.

\subsection{Post Treatment Efficacy within Study Groups}

Change from baseline was calculated by subtracting the baseline score from the post-treatment score of each visit.

An overall improvement in subjective and objective variables relatively to baseline was observed in the total study population. Tables 2, 3, 4 show the significant changes from baseline of each treatment group: E, DM and TP.

Table 1. Baseline characteristics of the study population before the treatments assignment in the three groups of study.

\begin{tabular}{|c|c|c|c|}
\hline Parameter & $\mathrm{E}(\mathrm{N}=40)$ & $\begin{array}{c}\mathrm{DM}(\mathrm{N}= \\
26)\end{array}$ & $\begin{array}{c}\mathrm{TP} \\
(\mathrm{N}=13)\end{array}$ \\
\hline Age & $5.61 \pm 0.51$ & $5.70 \pm 0.60$ & $6.63 \pm 0.93$ \\
\hline Males & 16 & 13 & 6 \\
\hline $\begin{array}{l}\text { TWEL_-uninvolved } \\
\left(\mathrm{g} \cdot \mathrm{m}^{-2} \cdot \mathrm{h}^{-1}\right)\end{array}$ & $17.49 \pm 8.51$ & $14.81 \pm 6.91$ & $15.92 \pm 3.88$ \\
\hline $\begin{array}{l}\text { TWEL-mild } \\
\left(\mathrm{g} \cdot \mathrm{m}^{-2} \cdot \mathrm{h}^{-1}\right)\end{array}$ & $24.86 \pm 16.34$ & $22.53 \pm 8.44$ & $21.22 \pm 8.99$ \\
\hline $\begin{array}{l}\text { TWEL-severe } \\
\left(\mathrm{g} \cdot \mathrm{m}^{-2} \cdot \mathrm{h}^{-1}\right)\end{array}$ & $32.00 \pm 16.37$ & $34.16 \pm 16.57$ & $32.11 \pm 14.51$ \\
\hline $\begin{array}{c}\mathrm{SCH} \text { - uninvolved } \\
\text { (AU) }\end{array}$ & $37.42 \pm 17.43$ & $36.53 \pm 9.64$ & $26.80 \pm 8.45$ \\
\hline $\mathrm{SCH}-\operatorname{mild}(\mathrm{AU})$ & $24.65 \pm 14.37$ & $23.86 \pm 8.86$ & $18.85 \pm 6.12$ \\
\hline $\mathrm{SCH}$-severe (AU) & $20.30 \pm 12.23$ & $19.58 \pm 10.16$ & $12.34 \pm 7.30$ \\
\hline BSA—total (\%) & $4.08 \pm 0.78$ & $6.12 \pm 1.41$ & $3.02 \pm 0.78$ \\
\hline $\begin{array}{c}\text { SCORAD } \\
\text { (Score points) }\end{array}$ & $36.35 \pm 13.19$ & $33.27 \pm 11.81$ & $43.58 \pm 7.97$ \\
\hline $\begin{array}{c}\text { OSAAD } \\
\text { (Score points) }\end{array}$ & $32.71 \pm 12.90$ & $36.91 \pm 12.47$ & $24.86 \pm 10.42$ \\
\hline IGA (Score points) & $2.52 \pm 1.48$ & $2.69 \pm 1.58$ & $1.89 \pm 1.08$ \\
\hline PGA (Score points) & $5.93 \pm 2.40$ & $6.50 \pm 2.76$ & $5.44 \pm 3.35$ \\
\hline
\end{tabular}

Assessment parameters (TEWL, SCH, BSA, SCORAD, OSAAD, IGA and $\mathrm{PGA})$ at the baseline was evaluated and recoreded. The data presented as MEAN of each study group \pm SEM.

Table 2. Efficacy assessment parameters-E group.

\begin{tabular}{ccc}
\hline Parameter & Week & Significance vs week 0 (p value) \\
\hline SCH-severe & 8,12 & $0.022,0.012$ \\
BSA-total & $2,4,6,8,12$ & $0.001,0.008,0.010,0.11,<0.001$ \\
SCORAD & $2,4,6,8,12$ & $<0.001,<0.001,, 0.001,<0.001,<0.001$ \\
IGA & $2,4,6,8,12$ & $<0.001,<0.001,0.001,<0.001,<0.001$ \\
PGA & $2,4,6,8,12$ & $<0.001,<0.001,, 0.001,<0.001,<0.001$ \\
\hline
\end{tabular}

Changes from baseline severity scores by treatment assignment in $\mathrm{E}$ group. The data presented as the significant $p$ values regarding the means between the first visit (week 0) and visits $2-6$.
Table 3. Efficacy assessment parameters-DM group.

\begin{tabular}{lcc}
\hline \multicolumn{1}{c}{ Parameter } & Week & Significance vs week $0(p$ value $)$ \\
\hline TEWL-severe & 6 & 0.025 (increase) \\
SCH-uninvolved & 12 & 0.024 \\
BSA total & $6,8,12$ & $0.014,0.042,0.032$ \\
OSAAD & 8,12 & $0.029,0.008$ \\
SCORAD & $2,4,6$, & $<0.001,<0.001$, \\
& 8,12 & $0.001,<0.001,<0.001$ \\
IGA & $4,6,8$, & $0.012,<0.001,<0.001,<0.001$ \\
& 12 & $0.001,0.002,0.001$, \\
PGA & $2,4,6$, & $<0.001,<0.001$ \\
\hline
\end{tabular}

Changes from baseline severity scores by treatment assignment in DM group. The data presented as the significant $\mathrm{p}$ values regarding the means between the first visit (week 0 ) and visits $2-6$.

Table 4. Efficacy assessment parameters-TP group.

\begin{tabular}{lcc}
\hline \multicolumn{1}{c}{ Parameter } & Week & $\begin{array}{c}\text { Significance vs week 0 } \\
\text { ( } \text { value) }\end{array}$ \\
\hline TEWL_uninvolved & $4,6,8$ & $0.006,0.009,0.039$ \\
SCH_uninvolved & $2,4,6$ & $0.009,0.027,0.019$ \\
SCH_mild & $2,4,6,8$ & $0.005,0.008$, \\
& & $<0.001,0.011$ \\
BSA total & $2,4,6,8,12$ & $0.001,0.004,0.004$, \\
OSAAD & $2,4,6$ & $0.007,0.016$ \\
SCORAD & $2,4,6,8,12$ & $0.009,0.005,0.004$ \\
& $2,4,6,8,12$ & $<0.001,<0.001$, \\
IGA & $2,4,12$ & $0.019,0.001,0.019,0.000 .001$ \\
PGA & & $0.020,0.024,0.001$ \\
\hline
\end{tabular}

Changes from baseline severity scores by treatment assignment in TP group. The data presented as the significant $p$ values regarding the means between the first visit (week 0 ) and visits $2-6$.

Treatment with E led to a significant increase in $\mathrm{SCH}$ of severe areas as well as a reduction in total affected BSA, SCORAD, IGA and PGA. No improvement had been detected in TEWL and OSAAD (Table 2).

Treatment with DM markedly decreased total affected BSA, OSAAD, SCORAD, IGA and PGA, but increased TEWL in severe areas at week 6 . It also significantly increased $\mathrm{SCH}$ in uninvolved areas (Table 3).

Treatment with TP significantly decreased TEWL of uninvolved areas, total affected BSA, OSAAD, SCORAD, IGA and PGA. It also significantly increased $\mathrm{SCH}$ in uninvolved and mild areas (Table 3).

\subsection{Post Treatment Efficacy between Study Groups}

Although each treatment group demonstrated an improvement in skin severity scores, treatment with $\mathrm{TP}$ mostly improved severity scores as compared to both $\mathrm{E}$ and DM groups: it showed a significant improvement in 


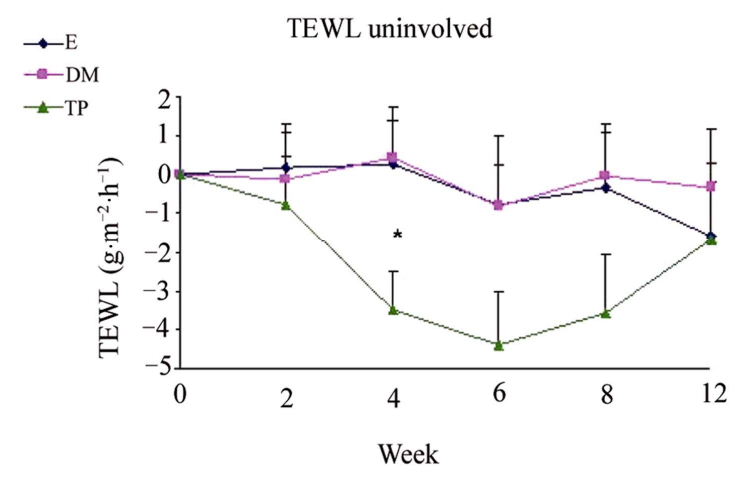

(a)

TEWL mild

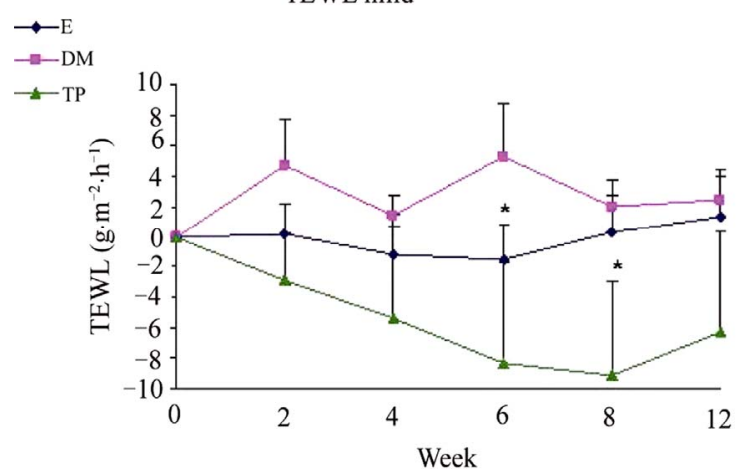

(b)

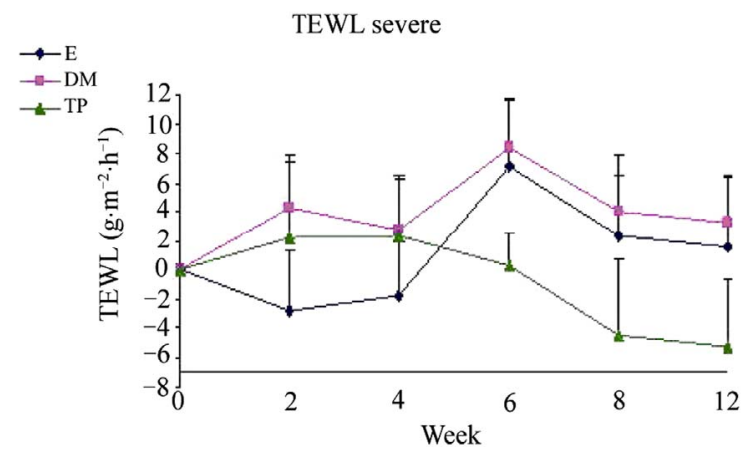

(c)

Figure 1. Changes from baseline scores of TEWL in uninvolved (a), mild (b) and severe (c) areas. Changes were calculated by subtracting the baseline score from the posttreatment scores of each visits (weeks $2,4,6,8,12$ ). Data is presented as MEAN \pm SEM. $* p<0.05$ TP vs DM.

SCH (Figure 2) and OSAAD (Figure 3(b)) scores compared to E group. TP also exhibited a significant improvement in TEWL (Figure 1), SCH (Figure 2) and OSAAD (Figure 3(b)) scores as compared to DM.

Treatment with E decreased more significantly IGA scores compared to TP (Figure 4(a)).

Differences in changes from baseline scores in BSA (Figure 3(a)), SCORAD (Figure 3(c)) and PGA (Figure 4(b)) were not significant between the all study groups.

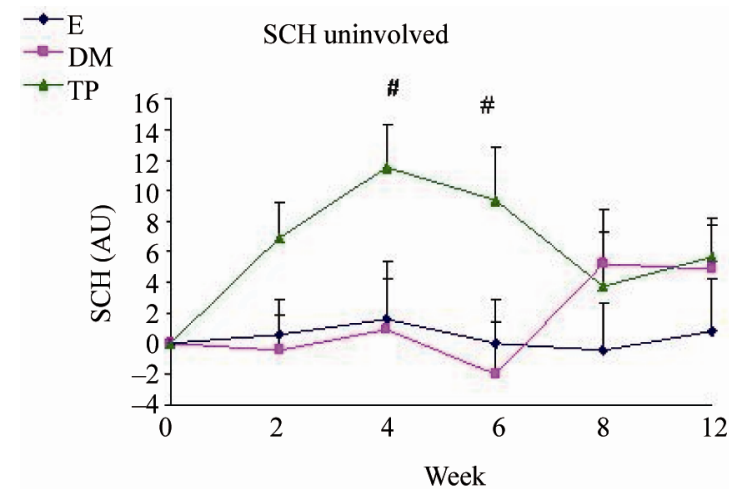

(a)

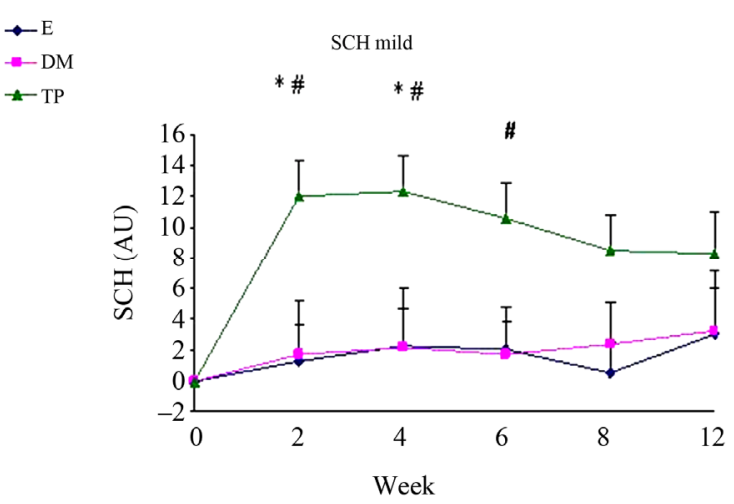

(b)

$\rightarrow \mathrm{E}$
$\rightarrow-\mathrm{DM}$
$\rightarrow \mathrm{TP}$

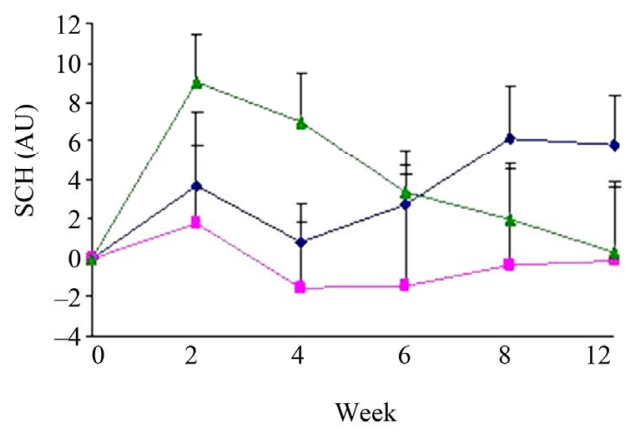

(c)

Figure 2. Changes from baseline scores of SCH in uninvolved (a), mild (b) and severe (c) areas. Changes were calculated by subtracting the baseline score from the posttreatment scores of each visits (weeks $2,4,6,8,12$ ). Data is presented as MEAN \pm SEM. ${ }^{*} p<0.05$ TP vs E; \#p $<0.05$ TP vs DM.

\section{Discussion}

$\mathrm{AD}$ is a barrier disease in which antigens and irritants might penetrate non-lesional skin, trigger or worsen the dermatitis and lead to infections. [27,28] Due to their delicate and vulnerable skin and defective barrier func- 


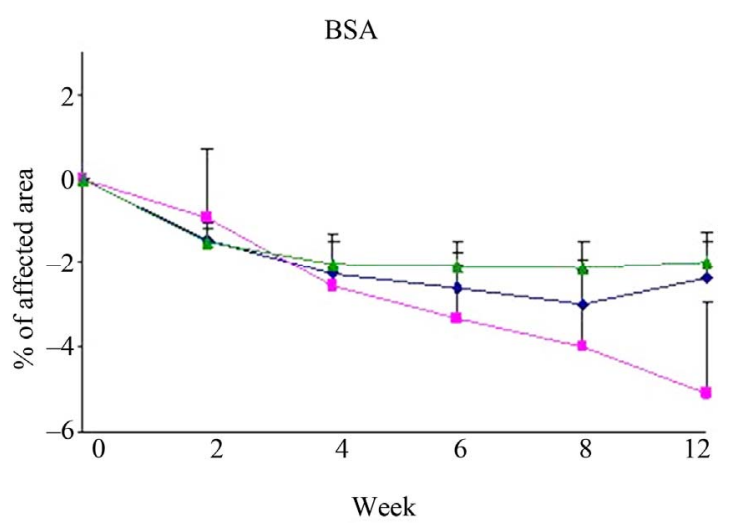

(a)

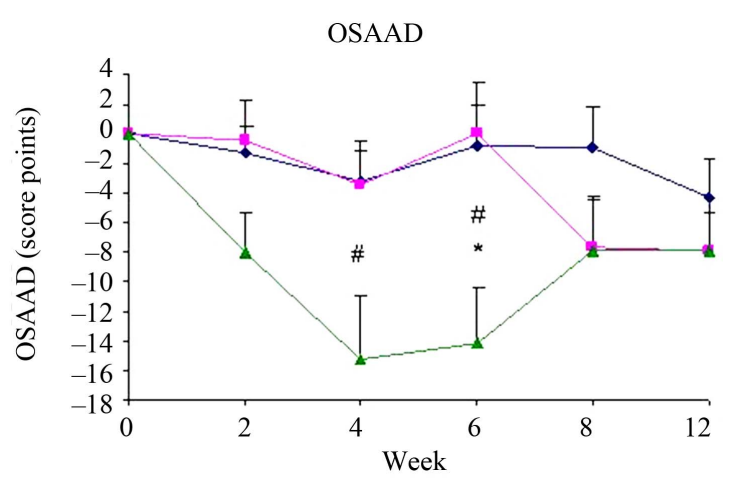

(b)

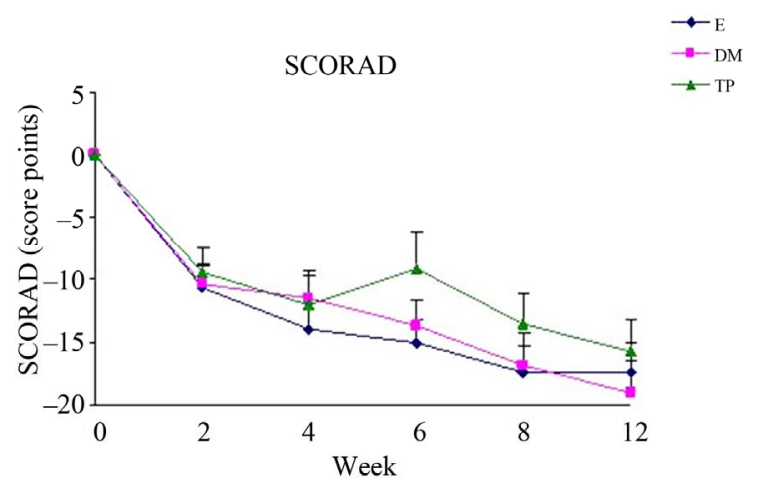

(c)

Figure 3. Changes from baseline scores of total BSA (a), OSAAD (b) and SCORAD (c). Changes were calculated by subtracting the baseline score from the post-treatment scores of each visits (weeks $2,4,6,8,12$ ). Data is presented as MEAN \pm SEM. ${ }^{*} p<0.05$ TP vs E; $\# p<0.05$ TP vs DM.

tion both in lesional and clinically uninvolved-appearing skin $[4,29]$, atopic children are exposed to higher risk of local adverse effects and systemic absorption through the epidermis [30]. Proksch et al. reported that bathing with solution of 5\% DS salts improved skin barrier function, $\mathrm{SCH}$, roughness and redness in adults AD patients [16]. In this study, we demonstrated a novel way of exploiting DS water by a leave-on skin preparation which might be

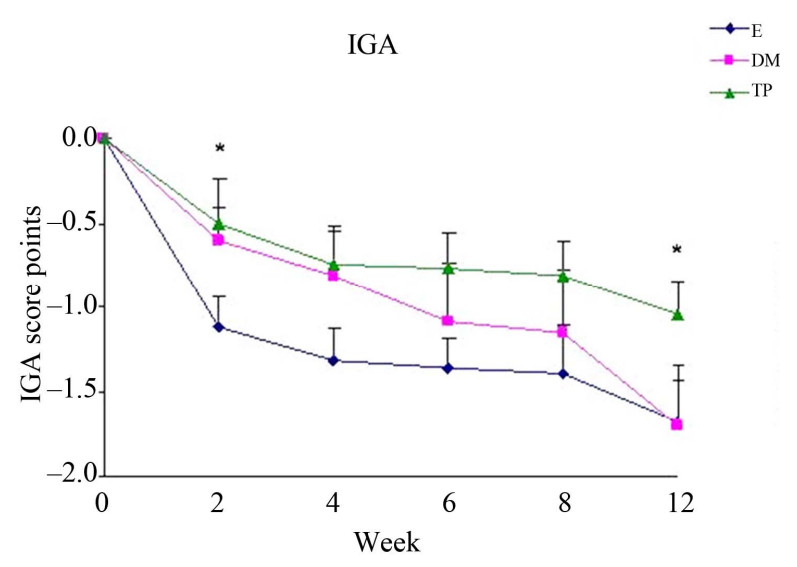

(a)
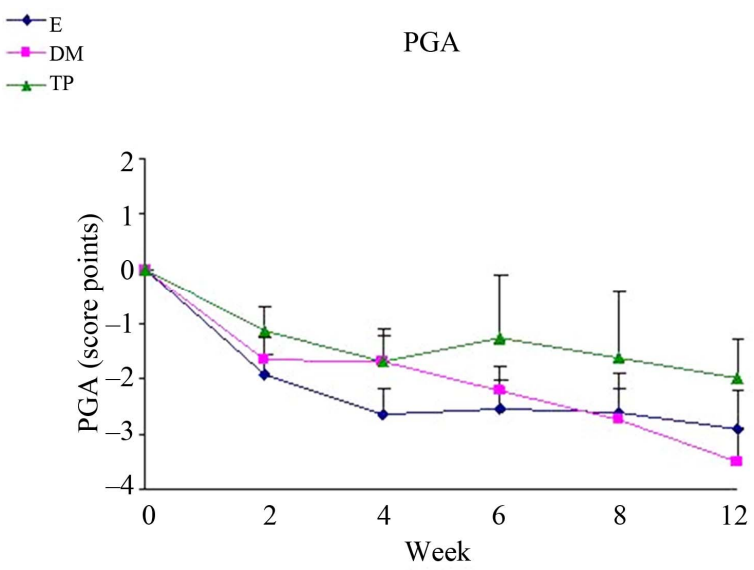

(b)

Figure 4. Changes from baseline scores of IGA (a) and PGA (b). Changes were calculated by subtracting the baseline score from the post-treatment scores of each visits (weeks 2, $4,6,8,12)$. Data is presented as MEAN \pm SEM. ${ }^{*} p<0.05$ TP vs E.

beneficial for children with AD. Both the concentrations of DS and delivery routes to the skin are different in these forms of treatment, thus the results may not be identical to mineral bathing.

Our result show the beneficial effects of leave on-skin emulsions enriched with DS minerals, especially in TP group, on skin barrier function and hydration. Taken together both analysis within and among groups regarding TEWL, SCH and OSAAD scores-TP significantly exhibited the most positive influence on skin (Figures 1, 2 and 3(b)). Moreover, at most time points of the treatment, TP application demonstrated the highest efficacy in AD severity indices as compared to the other creams.

The clinically normal, but dry and scaly uninvolved skin of AD patients seems to play a key role in relapses of the dermatitis. The cutaneous barrier function is generally impaired similarly in both non-lesional and lesional skin areas [28,31]. Considering this, it is notewor- 
thy that TP significantly reduced TEWL in uninvolvedappearing skin areas from week 4 . Treatment with TP also increased $\mathrm{SCH}$ compared to DM and E mostly in uninvolved and mildly affected areas. Furthermore, TP was superior in reducing the OSAAD score compared to the other tested treatments.

In most severity indices, TP stands out relatively to $\mathrm{DM}$ and $\mathrm{E}$. Thus, the observations concerning the clinical severity scores demonstrate significant benefits that can be attributed to DS water rather than DS mud.

It is notable that each one of the treatment regimens induced a significant improvement in the severity indices, as detected by total BSA, SCORAD, IGA and PGA (Figures 3(a)-(b), 4(a)-(b)). Regarding IGA scores, E showed a better decrease than TP (Figure 4(a)). Treatment with $\mathrm{E}$ also alleviated $\mathrm{SCH}$ in severe areas. The beneficial effects of all creams, including E, can be due to the involvement of active ingredients with anti-inflammatory properties, such as Bisabolol, Allantoin and Calendula extract [32]. Since among the treatment groups the efficacy of TP was the highest in most severity scores, it is possible that DS water play a key role regarding the improvement of skin condition. TP contains a higher concentration of DS water than DM and thus enriched with DS minerals. It can be speculated that DS minerals in this concentration might react with the other ingredients in a synergistic manner and thus, enhance the improvement efficacy. However, the action mechanism is unknown and this has to be further investigated.

The beneficial effect of DS water was reported also on aged human keratinocytes in cultures and human skin organ explants. This study demonstrated that the addition of DS water decreased the expression of some aging markers such as p16 and promoted proliferation by KI67. DS water also limited apoptotic damage after UVB irradiation [33].

Although DS minerals did not exhibit an added value concerning skin inflammation indices in comparison to non-mineral treatment, laboratory results on skin models support anti-inflammatory potential of both DM [22] and TP (unpublished data). The immune response in AD is Th2-mediated contributing to the high IgE levels $[4,5]$. Hence, it is possible that objective measurements for inflammation status regarding biochemical markers related to Th2, may demonstrate an improvement following application of DS minerals-containing creams.

In conclusion, we found that $\mathrm{AD}$ treatment with $\mathrm{TP}$, which is a DS water enriched body cream, markedly improved its severity scores. TP was more effective than DM and E concerning skin barrier function and skin hydration. Thus, TP can provide a maintenance therapy for AD patients. Further investigations should be employed in order to elucidate the effects of DS minerals containing creams on biochemical markers for inflammation, such as the levels of Th2-cytokines, IgE and eosinofils.

\section{REFERENCES}

[1] J. Callen, S. Chamlin, L. F. Eichenfield, et al., “A Systematic Review of the Safety of Topical Therapies for Atopic Dermatitis," British Journal of Dermatology, Vol. 156, No. 2, 2007, pp. 203-221. doi:10.1111/j.1365-2133.2006.07538.x

[2] D. Y. Leung and T. Bieber, "Atopic Dermatitis," The Lancet, Vol. 361, No. 9352, 2003, pp. 151-160. doi:10.1016/S0140-6736(03)12193-9

[3] M. Loden, A. C. Andersson and M. Lindberg, "Improvement in Skin Barrier Function in Patients with Atopic Dermatitis after Treatment with a Moisturizing Cream (Canoderm)," British Journal of Dermatology, Vol. 140, No. 2, 1999, pp. 264-267. doi:10.1046/j.1365-2133.1999.02660.x

[4] M. Breternitz, D. Kowatzki, M. Langenauer, P. Elsner and J. W. Fluhr, "Placebo-Controlled, Double-Blind, Randomized, Prospective Study of a Glycerol-Based Emollient on Eczematous Skin in Atopic Dermatitis: Biophysical and Clinical Evaluation," Skin Pharmacology and Physiology, Vol. 21, No. 1, 2008, pp. 39-45. doi:10.1159/000111134

[5] D. Y. Leung, "Atopic Dermatitis: New Insights and Opportunities for Therapeutic Intervention," Journal of Allergy and Clinical Immunology, Vol. 105, No. 5, 2000, pp. 860-876. doi:10.1067/mai.2000.106484

[6] I. Angelova-Fischer, A. Bauer, U. C. Hipler, et al., "The Objective Severity Assessment of Atopic Dermatitis (OSAAD) Score: Validity, Reliability and Sensitivity in Adult Patients with Atopic Dermatitis," British Journal of Dermatology, Vol. 153, No. 4, 2005, pp. 767-773. doi:10.1111/j.1365-2133.2005.06697.x

[7] E. A. Holm, H. C. Wulf, L. Thomassen and G. B. Jemec, "Instrumental Assessment of Atopic Eczema: Validation of Transepidermal Water Loss, Stratum Corneum Hydration, Erythema, Scaling, and Edema," Journal of the American Academy of Dermatology, Vol. 55, No. 5, 2006, pp. 772-780. doi:10.1016/j.jaad.2006.03.036

[8] J. L. Sugarman, J. W. Fluhr, A. J. Fowler, T. Bruckner, T. L. Diepgen and M. L. Williams, "The Objective Severity Assessment of Atopic Dermatitis Score: An Objective Measure Using Permeability Barrier Function and Stratum Corneum Hydration with Computer-Assisted Estimates for Extent of Disease," Archives of Dermatology, Vol. 139, No. 11, 2003, pp. 1417-1422.

doi:10.1001/archderm.139.11.1417

[9] S. Halevy and S. Sukenik, "Different Modalities of Spa Therapy for Skin Diseases at the Dead Sea Area," Archives of Dermatology, Vol. 134, No. 11, 1998, pp. 14161420. doi:10.1001/archderm.134.11.1416

[10] E. Hodak, A. B. Gottlieb, T. Segal, et al., "Climatotherapy at the Dead Sea is a Remittive Therapy for Psoriasis: Combined Effects on Epidermal and Immunologic Activation," Journal of the American Academy of Dermatology, Vol. 49, No. 3, 2003, pp. 451-457. 
doi:10.1067/S0190-9622(03)00916-2

[11] S. W. Moses, M. David, E. Goldhammer, A. Tal and S. Sukenik, "The Dead Sea, a Unique Natural Health Resort," The Israel Medical Association Journal, Vol. 8, No. 7, 2006, pp. 483-488.

[12] A. Deters, E. Schnetz, M. Schmidt and A. Hensel, "Effects of Zinc Histidine and Zinc Sulfate on Natural Human Keratinocytes," Forsch Komplementarmed Klass Naturheilkd, Vol. 10, No. 1, 2003, pp. 19-25. doi:10.1159/000069903

[13] Z. Ma'or, Y. Henis, Y. Alon, E. Orlov, K. B. Sorensen and A. Oren, "Antimicrobial Properties of Dead Sea Black Mineral Mud," International Journal of Dermatology, Vol. 45, No. 5, 2006, pp. 504-511. doi:10.1111/j.1365-4632.2005.02621.x

[14] M. Denda, C. Katagiri, T. Hirao, N. Maruyama and M. Takahashi, "Some Magnesium Salts and a Mixture of Magnesium and Calcium Salts Accelerate Skin Barrier Recovery," Archives of Dermatological Research, Vol. 291, No. 10, 1999, pp. 560-563. doi:10.1007/s004030050454

[15] C. M. Schempp, H. C. Dittmar, D. Hummler, et al., "Magnesium Ions Inhibit the Antigen-Presenting Function of Human Epidermal Langerhans Cells in Vivo and in Vitro. Involvement of ATPase, HLA-DR, B7 Molecules, and Cytokines," Journal of Investigative Dermatology, Vol. 115, No. 4, 2000, pp. 680-686. doi:10.1046/j.1523-1747.2000.00090.x

[16] E. Proksch, H. P. Nissen, M. Bremgartner and C. Urquhart, "Bathing in a Magnesium-Rich Dead Sea Salt Solution Improves Skin Barrier Function, Enhances Skin Hydration, and Reduces Inflammation in Atopic Dry Skin," International Journal of Dermatology, Vol. 44, No. 2, 2005, pp. 151-157. doi:10.1111/j.1365-4632.2005.02079.x

[17] M. Iwata, T. Takebayashi, H. Ohta, R. E. Alcalde, Y. Itano and T. Matsumura, "Zinc Accumulation and Metallothionein Gene Expression in the Proliferating Epidermis during Wound Healing in Mouse Skin," Histochemistry and Cell Biology, Vol. 112, No. 4, 1999, pp. 283-290. doi: $10.1007 / \mathrm{s} 004180050449$

[18] A. Nissenbaum, J. Rullkotter and Y. Yechieli, "Are the Curative Properties of 'Black Mud' from the Dead Sea Due to the Presence of Bitumen (Asphalt) or Other Types of Organic Matter?" Environ Geochemistry Health, Vol. 24, No. 4, 2002, pp. 327-335. doi:10.1023/A:1020559717754

[19] W. Diezel, E. Schulz, J. Laskowski, et al., "Magnesium Ions: Topical Application and Inhibition of the Croton Oil-Induced Inflammation," Zschr. Hautkrh, Vol. 69, 1994, pp. 759-760.

[20] J. Shani, S. Barak, D. Levi, et al., "Skin Penetration of Minerals in Psoriatics and Guinea-Pigs Bathing in Hypertonic Salt Solutions," Pharmacology Research Communications, Vol. 17, No. 6, 1985, pp. 501-512. doi:10.1016/0031-6989(85)90123-7

[21] M. Speich and B. Bousquet, "Magnesium: Recent Data on Metabolism, Exploration, Pathology and Therapeu- tics," Magnesium-Bulletin, Vol. 13, No. 4, 1991, pp. 116121.

[22] M. Portugal-Cohen, Y. Soroka, Z. Ma'or, et al., "Protective Effects of a Cream Containing Dead Sea Minerals against UVB-Induced Stress in Human Skin," Experimental Dermatology, Vol. 18, No. 9, 2009, pp. 781-788. doi:10.1111/j.1600-0625.2009.00865.x

[23] J. M. R. G. Hanifin, "Diagnostic Features of Atopic Dermatitis," Acta Dermato-Venereologica, Vol. 92, 1980, pp. 44-47.

[24] V. Rogiers, "EEMCO Guidance for the Assessment of Transepidermal Water Loss in Cosmetic Sciences," Skin Pharmacology and Applied Skin Physiology, Vol. 14, No. 2, 2001, pp. 117-128. doi:10.1159/000056341

[25] E. Berardesca, J. L. Leveque and P. Masson, "EEMCO Guidance for the Measurement of Skin Microcirculation," Skin Pharmacology and Applied Skin Physiology, Vol. 15, No. 6, 2002, pp. 442-456. doi:10.1159/000066451

[26] Anonym, "Severity Scoring of Atopic Dermatitis: The SCORAD Index. Consensus Report of the European Task Force on Atopic Dermatitis," Dermatology, Vol. 186, No. 1, 1993, pp. 23-31.

[27] M. J. Choi and H. I. Maibach, "Role of Ceramides in Barrier Function of Healthy and Diseased Skin," American Journal of Clinical Dermatology, Vol. 6, No. 4, 2005, pp. 215-223. doi:10.2165/00128071-200506040-00002

[28] H. Matsuki, K. Kiyokane, T. Matsuki, S. Sato and G. Imokawa, "Recharacterization of the Nonlesional Dry Skin in Atopic Dermatitis through Disrupted Barrier Function," Exogenous Dermatology, Vol. 3, No. 6, 2004, pp. 282-292. doi:10.1159/000091909

[29] E. Proksch, J. M. Jensen and P. M. Elias, "Skin Lipids and Epidermal Differentiation in Atopic Dermatitis," Clinics in Dermatology, Vol. 21, No. 2, 2003, pp. 134-144. doi:10.1016/S0738-081X(02)00370-X

[30] D. Staab, D. Pariser, A. B. Gottlieb, et al., "Low Systemic Absorption and Good Tolerability of Pimecrolimus, Administered as $1 \%$ Cream (Elidel) in Infants with Atopic Dermatitis-A Multicenter, 3-Week, Open-Label Study," Pediatric Dermatology, Vol. 22, No. 5, 2005, pp. 465-471. doi:10.1111/j.1525-1470.2005.00128.x

[31] M. Hata, Y. Tokura, M. Takigawa, et al., "Assessment of Epidermal Barrier Function by Photoacoustic Spectrometry in Relation to Its Importance in the Pathogenesis of Atopic Dermatitis," Laboratory Investigation, Vol. 82, No. 11, 2002, pp. 1451-1461.

[32] P. Pommier, F. Gomez, M. P. Sunyach, A. D'Hombres, C. Carrie and X. Montbarbon, "Phase III Randomized Trial of Calendula Officinalis Compared with Trolamine for the Prevention of Acute Dermatitis during Irradiation for Breast Cancer," Journal of Clinical Oncology, Vol. 22, No. 8, 2004, pp. 1447-1453. doi:10.1200/JCO.2004.07.063

[33] Y. Soroka, Z. Ma'or, Y. Leshem, et al., "Aged Keratinocyte Phenotyping: Morphology, Biochemical Markers and Effects of Dead Sea Minerals," Exogenous Dermatology, Vol. 43, No. 10, 2008, pp. 947-957. doi:10.1016/j.exger.2008.08.003 